\title{
A Robust Functional Minimization Technique to Protect Image Details from Disturbances
}

\author{
Md. Robiul Islam ${ }^{1}$ \\ ${ }^{1}$ College of Computer Science and Software Engineering, Shenzhen University, Shenzhen 518060, China \\ E-mail: robi@szu.eddu.cn \\ Chen $\mathrm{Xu}^{2^{*}} \mathrm{Yu} \mathrm{Han}^{2}$, Sanjida Sultana Putul ${ }^{3}$ and Rana Aamir Raza ${ }^{1}$ \\ ${ }^{2}$ College of Mathematics and Computational Science, Shenzhen University, Shenzhen 518060, China \\ ${ }^{3}$ Department of Computer Science and Engineering, North Western University, Khulna, Bangladesh \\ E-mail: $\left\{{ }^{*}\right.$ xuchen_szu, hany, aamir $\} @$ szu.edu.cn, ssputul@gmail.com
}

Received: 03 March 2019; Accepted: 21 April 2019; Published: 08 July 2019

\begin{abstract}
Image capturing using faulty systems or environmental vulnerabilities always degrade the image quality and causes the distortion of true details from the original imaging signals. Thus a robust way of image enhancement and edge preservation is an enormously requirement for smooth imaging operations. Although, many techniques have been deployed in this area during the decades for its betterment. However, the key challenges are remain towards better tradeoff between image enhancement and details protection. Therefore, this study inspects the existing limitations and proposes a robust technique based on functional minimization scheme in variational framework for ensuring better performance in case of image enhancement and details preservation simultaneously. A vigorous way to solve the minimization problem is also develop to make sure the efficiency of the proposed technique than some other traditional techniques.
\end{abstract}

Index Terms - Image enhancement, dual projection, total variation regularizer, functional minimization.

\section{INTRODUCTION}

During the last decades, imaging activities all over the world have been increasing tremendously due to the huge application of cameras, videos or other imaging instruments in many areas of application. All most in every sectors including medical science, computer vision, satellite imaging, classification or segmentation, motion detection, fraud or intrusion prevention, traffic security or road safety where usages of images are overwhelming. Due to the large volume of image applications, it is an emerging issue how an image can be smoothed while preserving its own and actual properties. Nowadays, it is not an issue how much the imaging equipment or video capturing instrument is efficient; however, the captured images or videos are always found in less or more erroneous condition due to the different disturbances during its transmission or acquisition. A faulty imaging system always hampers image quality by incurring unpredicted noises to the original imaging signals. The erroneous images subsequently lead malfunctioning during image operation or analysis and produce garbage output. Therefore, effective and efficient way of image smoothing, as well as developing a robust technique to remove noise and preserving image properties is appreciative and still challenging for the community of image vision and analysis. In real applications, image smoothing is always a noteworthy issue since its preprocessing is urgent to construct a good quality image. In the real field of image applications, it is observed that the bulk amount of noises is incurred from different known and unknown sources during its capturing phase. Therefore, an attempt to eliminate these impurities from the contaminated images is unquestionably necessary to produce a clean version that would be more useful in any image related operations.

During a plenty of times, many approaches for the purpose have been discovered in scientific literature. However, the image smoothing problems significantly come into the notice are reported in many research articles including local filtering-based image smoothing $[1,2]$, nonlocal-based methods [3- 6] are also noted in literature. Nonlocal wavelet-based method [7, 8], nonlocal-based sparse coding strategy [9], nonlocal lowrank [10], the sparse representation techniques [11], shearlet-based model [12], curvelet-based method [13], dictionary-based approaches [14, 15], soft-thresholding method [16], image deblurring technique using regularization [17], the radial basis function (RBF)-based method [18] and image retrieval with color and angle representation [19] are also remarkable in applications. However, many other methods based on variation and partial differential equation (PDE) have been proposed widely since variational calculus come out recently as a powerful tool for image-smoothing and model solutions. Moreover, the solution of the models can ensure image smoothness and details preservation abilities, those abilities are also reported as in the PDE-based methods $[20,22]$. Similarly, energy minimization or variationalbased approaches such as [23- 25, 27-29] are widely 
using in image enhancement problems. The nonconvexbased smoothing approaches such as [30-33] can also preserve image details. However, a total variation (TV)based scheme known as ROF technique [34] and the Chamboll's method [35] in the form of minimization problems are famous for regularizing noisy images. An efficient impulse noise removal approach is described by $\mathrm{Yu}$ et al. [36]. In this approach, the time for denoising process is relatively less as their algorithm iteratively implemented, however the performance is not good enough. A fast minimization method for image restoration is proposed by Nikolova et. al. [31]. They used nonconvex non-smooth regularization on L2 norm to show the advantages over convex regularization with edges preservation ability. However, the technique is computationally inefficient since it requires a nonconvex smoothing. In the same direction, Zuo et al. [32] proposed an adaptive nonconvex non-smooth (ANCNS) regularization approach to show better restoration of image edges, however it incurred time penalty as it requires a nonconvex minimization. In [37], Sutour et al. proposed a new method with TV regularizer and nonlocal means scheme to reduce the residual noise but the method still unknown to deal with other types of regularizers except TV-based regularizer. In [26], Liu et al. proposed a higher order total variational (HDTV) model as the form of weighted L1-L2 mixed norms for image enhancement but the convergence of HDTV is still unknown in this work. In [21], Wang et al. proposed a nonlinear fourth-order PDE based denoising method by approximating energy to preserve textures only for additive noise but the performance still unknown for other types of noises.

The existing literature reviews reveal many imageenhancement techniques those have been introduced for endeavoring image quality. However, intensive analysis is still enduring toward superior way of preserving image details and noise removal simultaneously. Although some of the existing techniques perform less or more effective in some context, but still there are many techniques are not good enough. For example, the TV-based image enhancement technique [34] uses convex energy to protect image properties. However, the high isotropic polishing phenomena hamper some important image details. To defeat this problem, a nonconvex way of enhancement is proposed in [27, 30] which ensure the ability of details protection and noise elimination. However, it is much slower method and cannot guarantee unique solution. The technique also recognizes noises as image element in homogeneous regions. As a result, a new technique is reasonably necessary to remove noise while keeping the original image properties. Therefore, the objective of this work is to introduce a robust functional minimizing technique for better image smoothing performance while keeping the actual image details.

Rest of the paper is separated as follows: Section II describes the details of proposed technique including the basic image enhancement consequences. The Section III shows experimental studies. Performance assessment is reported in Section IV. Finally, Section V concludes this paper.

\section{Details of Proposed TeChnique}

During the last decades, imaging activities all over the world have been increasing tremendously due to the huge application of cameras, videos or other imaging instruments in many areas of application. Generally, faulty imaging systems, environmental problems or distortions during capturing process often degrade image quality by incurring enormous noises. Eliminating these susceptible artifacts from the original images is the vital requirement for smoothing and recovering the image details. For the purpose, the key concern in image smoothing is to protect details while eliminating noise or other vulnerable objects. A good balance between noise elimination and details protection is extremely necessary for constructing high quality images. Therefore, we attempt to investigate the existing limitations and aim towards a robust way of image quality improvement using a new methodology as describe in the subsequent section.

Noise reduction simply refers to the way of eliminating disturbance-artifacts from the actual images. Let $p_{i j}$ is the real or authentic image where $p: \Omega \subset R^{2} \rightarrow R$ and $\Omega$ is image domain, $q_{i j}$ is the noisy image with Gaussian noise $\eta_{i j}$, then mathematically, the degraded image can model as Eq. (1):

$$
q_{i j}=p_{i j}+\eta_{i j}
$$

The reconstruction of $p_{i j}$ from $q_{i j}$ here is the ultimate objective. An approximation to $p_{i j}$ can be ensure from the following minimization problem (2):

$$
\min \int_{\Omega}\left|q_{i j}-p_{i j}\right|^{2}
$$

To manage the ill-posed issue [38] in minimization, an image regularization or smoothing term as in Eq. (3) is essential with the original signal to resolve the minimization problems (2):

$$
\min _{p}\left\{\begin{array}{l}
L(p)=F(p) \\
=\int_{\Omega}\left|q_{i j}-p_{i j}\right|^{2} d x \\
+\lambda \int_{\Omega}\left|\nabla p_{i j}\right|^{2} d x
\end{array}\right\}
$$

In (3), the first and second terms treat as fidelity and the regularizer or smoothing term respectively. In TVbased image restoration [34], the fidelity and regularization terms describes the noise levels and image 
smoothness respectively. In practice, the image enhancement performance largely depends on smoothing terms. In (3) second term regularizes the images where $\lambda$ is the balancing parameter. The dilemma (3) can be flourished by Euler-Lagrange Equation as (4) which pledge a unique solution:

$$
p_{i j}-q_{i j}-\lambda \Delta p_{i j}=0
$$

with boundary conditions:

$$
\frac{\delta p}{\delta D}=0 \text { on } \Omega \text { (D indicates the outward normal to } \Omega \text { ) }
$$

and the necessary mathematical propositions to solve the problems are discussed below:

The Gradient of $p$ refers to $\nabla p$ as the gradient vector, which can be defined as:

$$
\nabla p(x)=\left(\frac{\partial p}{\partial x_{1}}, \ldots, \frac{\partial p}{\partial x_{n}}\right) \in \mathbb{R}^{n}
$$

The divergence on $\nabla p$ as the following terminology:

$$
\operatorname{div}(\nabla p)=\sum_{i=1}^{n} \frac{\partial p}{\partial x_{i}}
$$

The Laplace $p(\Delta p)$ can be expressed as:

$$
\Delta p=\operatorname{div}(\nabla p)=\sum_{i=1}^{n} \frac{\partial^{2} p}{\partial x_{i}^{2}}
$$

The TV-based technique [34] utilizes $L^{1}$ norm of $\nabla p_{i j}$ in (4) instead of $L^{2}$ in (3), and it express as in the form of Eq. (5):

$$
\int_{\Omega}\left|q_{i j}-p_{i j}\right|^{2} d x+\lambda \int_{\Omega}\left|\nabla p_{i j}\right| d x
$$

where the term $\int_{\Omega}\left|\nabla p_{i j}\right|$ in (5) stands for the total variation (TV) of $p_{i j}$ image. For implementation, we need to introduce a discrete form of TV-based approach as in the following way. If the minimization problem of discrete functional $F$ then:

$$
\begin{aligned}
\min F & =\min \sum_{i, j} L\left(p_{i j}-q_{i j}\right) \\
& =\min \sum_{i, j} \frac{\lambda}{2}\left|p_{i j}-q_{i j}\right|^{2}+\left|\nabla p_{i j}\right|
\end{aligned}
$$

where $\left|\nabla p_{i j}\right|=\sqrt{\left(\Delta_{x} p_{i j}\right)^{2}+\left(\Delta_{y} p_{i j}\right)^{2}}$

$$
\Delta_{x} p_{i j}=p_{i+1, j}-p_{i j} \text { and } \Delta_{y} p_{i j}=p_{i, j+1}-p_{i j}
$$

Although TV-based method can enhance images, however it eliminates some valuable image details simultaneously due to its polishing effects. Thus, an alternative regularizing term for the quality solution is introduced in [27, 30] and illustrate the efficiency of this nonconvex technique as (6):

$$
\int_{\Omega}\left|q_{i j}-p_{i j}\right|^{2} d x+\int_{\Omega} \mu\left(\left|\nabla p_{i j}\right|\right) d x
$$

The function $\mu(r)=\frac{\beta r}{1+\beta r}$ where $\beta$ is a positive parameter. The vital advantage of this nonconvex technique (6) is the ability of details preservation but alarming issues are uncertainty of unique solution and allow noise as original detail particularly in boundary regions. As a remedy, we examine the respective limitations and propose a robust functional minimizing technique that can be modeled as in the following form of functional (7):

$$
\begin{gathered}
L(p)=\frac{1}{2} \int_{\Omega}\left|q_{i j}-p_{i j}\right|^{2} d x+\delta \int_{\Omega}\left|\nabla p_{i j}\right| d x \\
+(1-\delta) \int_{\Omega} \mu\left(\left|\nabla p_{i j}\right|\right) d x
\end{gathered}
$$

The functional (7) can ensure the better noise reduction and edge protection ability simultaneously since it inherits the benefits of both TV and nonconvex-based techniques by assigning $\delta=1$ and $\delta=0$ respectively. The use of discrete variational principle to the discrete functional is necessary to solve the minimization problem. The appropriate parameters and weight estimation always influence the better trade-off during image smoothing process. However, the direct solution of (7) is obscured due to nonconvex functional. Therefore, we need to handle the image regularizer i.e. handling smoothing term by iteratively reweighted method as (8):

$$
\mu\left(\left|\nabla p_{i j}\right|\right)=\left(\frac{1+\xi}{1+\xi\left|\nabla p_{i j}\right|}\right)\left|\Delta p_{i j}\right|=\psi\left|\Delta p_{i j}\right|
$$

$$
\text { In (8), we define a robust weight } \psi \text { as: } \frac{1+\xi}{1+\xi\left|\nabla p_{i j}\right|}
$$

where $\xi$ is the arbitrary positive parameter ranges [0.01, 0.09]. For eliminating noise and protecting image details to construct visually high quality image, our robust technique (8) can be implemented by solving the following functional minimization problem (9):

$$
\min _{p}\left\{\begin{array}{l}
\frac{1}{2} \int_{\Omega}|q-p|^{2} d x \\
+\int_{\Omega}(\delta+(1-\delta) \psi)|\nabla p| d x
\end{array}\right\}
$$


As $\psi$ is given, the above Eq. (9) is convex and unique solution is confirmed. The Chambolle's projection algorithm [35] is used to attain the solution of the functional minimization (9), which is specified as Eq. (10):

$$
p_{i}=q_{i}-\lambda \operatorname{div} \mathbf{z}_{i}
$$

where $\operatorname{div} \mathbf{z}$ is the divergence (div) of the vector $\mathbf{z}: \Omega \rightarrow \mathfrak{R} \times \mathfrak{R}$ expresses a vector function and $\mathbf{z}_{i} \in \mathfrak{R}^{n}$ is selected by a fixed point principle : given $\mathbf{z}^{0}=0$ is a first estimate and suppose time-step $=\sigma$, then we iterate the scheme (11) for the solutions:

$$
\mathbf{z}_{i}^{t+1}=\frac{\mathbf{z}_{i}^{t}+\sigma \nabla\left(\operatorname{div} \mathbf{z}_{i}^{t}-q_{i}\right)}{1+\left(\frac{\sigma}{\delta}+(1-\delta) \psi\left|\operatorname{div} \mathbf{z}_{i}^{t}-q_{i}\right|\right)}
$$

\section{EXPERIMENTAL STUDIES}

To verify the effectiveness of diverse image enhancement techniques, the experimental details are presented in this section. Matlab 2017a on a computer configured with core-i5 and $3.20 \mathrm{GHz}$ is used for implementing the algorithms. For experimental setup, the parameters $\lambda, \delta, \sigma$ and $\xi$ are required to be initialized before execute the experiments. The standardize image size is $[256 \times 256]$ and range of intensity is $[1,256]$ respectively. The controlling parameter $\lambda$ is equivalent to the noise level and $\delta$ is selected from a fixed range $[0,0.6]$ as in $[27,30]$, however we choose $\delta$ for any values from the range $[0,1]$. In experiment, the parameters $\lambda$ and $\delta$ are for the role of controlling the smoothness. We set $\sigma$ for any random value from the ranges $[0<\sigma<1]$. The value of $\xi$ is determined from the random ranges $[0.01,0.09]$. To assess the performance, a set of reference clean images in (a) and their corresponding noisy images in (b) of Fig.1 are used respectively. The parameter $N$ is connected to the standard deviation of the noise $\eta$. To condense the pages of the paper, the noise parameters $N$ is fixed from the set $\{10,20,30,40\}$ where higher $N$ specify stronger noise intensity. However, any intervals of noise intensity can be tested for the experiments.

\section{PERformance Assesments}

The performance comparisons can be validated by both visual and numerical improvements. Based on original and restored images, signal to noise ratio (SNR), peak signal to noise ratio (PSNR) and structural similarity (SSIM) can be the evaluation indicators. The qualitative indicator SSIM is used for image quality comparisons between proposed and existing techniques. There are huge numbers of reference images have been used for performance assessment in this domain. However, we use several images for experimental analysis and quality evaluation as it facilitates to minimize the length of the paper, it does not mean the limitation of diversified using of other images.

The SSIM index is calculated based on the similarity between the clean image and restored image. The vale between the range 0 and 1 is used as the SSIM index. The high value of SSIM index signifies more structure similarity between the original image and resorted image. The SSIM is measured as Eq. (12) based on clean image $p$ and reconstructed image $\bar{p}$ :

$$
\operatorname{SSIM}=\frac{\left(2 \beta_{p} \beta_{\bar{p}}+\theta_{1}\right)\left(2 \phi_{p \bar{p}}+\theta_{2}\right)}{\left(\beta_{p}^{2}+\beta \frac{2}{p}+\theta_{1}\right)\left(\phi_{p}^{2}+\phi_{\bar{p}}^{2}+\theta_{2}\right)}
$$

where $\beta_{p}$ and $\beta_{\bar{p}}$ are the mean values of the clean and restored image, respectively. Similarly, $\phi_{p}$ and $\phi_{\bar{p}}$ are the variance values of the clean and restored image, respectively. $\phi_{p \bar{p}}$ is the covariance of images $p$ and $\bar{p}$; two variables $\theta_{1}$ and $\theta_{2}$ are assigned with a weak denominator to stabilize the division.

We exhibit image smoothing results including image details preservation ability in (c)-(e) of Fig.1 for visual assessment, and the results in Table 1 for quantitative assessment respectively. From the Table 1, it is observed that our proposed technique on average attain higher SSIM values than the other traditional techniques. Evaluating the results in (c)-(e) of Fig.1, it can make sure that the proposed technique can wipe out much noise and protect image true details more competently, particularly in edge regions. For example, the smoothed results in (c) of Fig.1 are generated by TV-based technique still take hold of visible noise and produce over enhancement while iteration increased. The images by nonconvexbased technique are revealed in (d) of Fig.1. The results show that some image details are also removed in (d) and over-smoothed in (c) than the results exposed in (e) of Fig.1.

For more precise comparisons, we enlarged restored images in (c)-(e) of Fig.1. From the magnified observation, the restored images by proposed technique in (e) make sure the visually better quality images than the same images restored by TV-based and nonconvexbased smoothing technique as shown (c) and (d) in Fig.1 respectively. In (e) of Fig.1, the restored boundary objects or edges are much clean and sharper with more scene details. However, images (c) still contain noises and oversmoothed the boundary areas; and images (d) restore noises with image details in homogeneous regions. It indicates that the proposed technique simultaneously can remove more noise in homogeneous regions and preserve edge details efficiently than other methods. 
$\mathbf{a}$



b

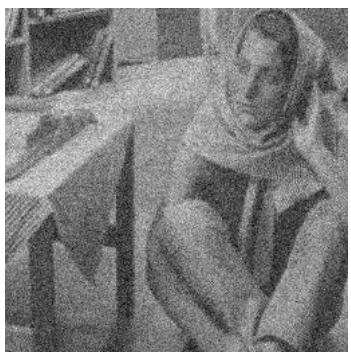

c

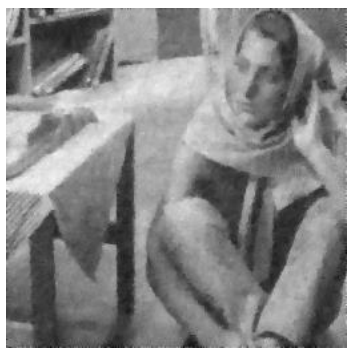

d



e

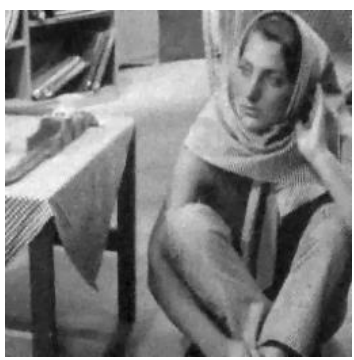

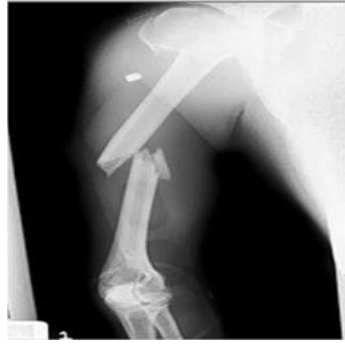


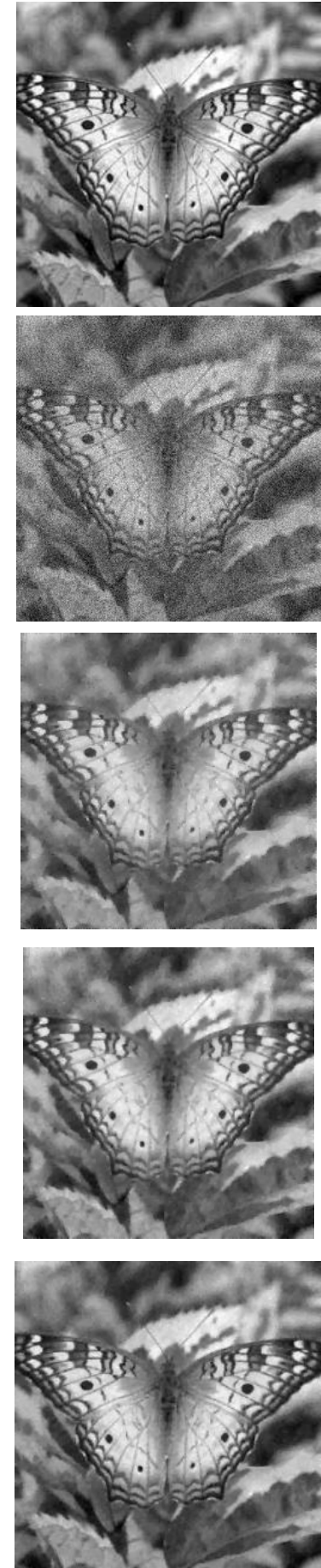

Fig.1. Performance comparisons: a original image, $\mathbf{b}$ noised image with $\mathrm{N}=30$, $\mathbf{c}$ TV-based technique $\mathbf{d}$ Nonconvex-based technique and e proposed technique 
Table 1. SSIM comparisons and performance evaluation for different techniques

\begin{tabular}{|c|c|c|c|c|c|}
\hline Name & $\mathbf{N}$ & Noisy Image & TV-based (5) & Nonconvex-based (6) & Proposed \\
\hline \multirow{4}{*}{ House } & 10 & 0.611 & 0.829 & 0.854 & 0.867 \\
\hline & 20 & 0.501 & 0.706 & 0.738 & 0.743 \\
\hline & 30 & 0.409 & 0.635 & 0.663 & 0.671 \\
\hline & 40 & 0.365 & 0.593 & 0.612 & 0.622 \\
\hline \multirow{4}{*}{ Peppers } & 10 & 0.631 & 0.899 & 0.919 & 0.933 \\
\hline & 20 & 0.559 & 0.839 & 0.863 & 0.871 \\
\hline & 30 & 0.514 & 0.794 & 0.817 & 0.830 \\
\hline & 40 & 0.466 & 0.756 & 0.779 & 0.798 \\
\hline \multirow{4}{*}{ Butterfly } & 10 & 0.663 & 0.896 & 0.920 & 0.927 \\
\hline & 20 & 0.591 & 0.821 & 0.846 & 0.853 \\
\hline & 30 & 0.497 & 0.761 & 0.785 & 0.792 \\
\hline & 40 & 0.399 & 0.713 & 0.736 & 0.741 \\
\hline \multirow{4}{*}{ Cat } & 10 & 0.626 & 0.879 & 0.894 & 0.907 \\
\hline & 20 & 0.538 & 0.797 & 0.817 & 0.831 \\
\hline & 30 & 0.478 & 0.736 & 0.760 & 0.775 \\
\hline & 40 & 0.397 & 0.698 & 0.718 & 0.722 \\
\hline \multirow{4}{*}{ Flower } & 10 & 0.619 & 0.887 & 0.908 & 0.925 \\
\hline & 20 & 0.583 & 0.833 & 0.858 & 0.865 \\
\hline & 30 & 0.506 & 0.789 & 0.812 & 0.821 \\
\hline & 40 & 0.498 & 0.754 & 0.774 & 0.791 \\
\hline \multirow{4}{*}{ Barbara } & 10 & 0.632 & 0.869 & 0.884 & 0.891 \\
\hline & 20 & 0.528 & 0.792 & 0.809 & 0.817 \\
\hline & 30 & 0.493 & 0.741 & 0.760 & 0.769 \\
\hline & 40 & 0.391 & 0.701 & 0.723 & 0.734 \\
\hline \multirow{4}{*}{ Carpet } & 10 & 0.679 & 0.934 & 0.959 & 0.975 \\
\hline & 20 & 0.586 & 0.848 & 0.876 & 0.919 \\
\hline & 30 & 0.491 & 0.752 & 0.789 & 0.853 \\
\hline & 40 & 0.397 & 0.693 & 0.714 & 0.793 \\
\hline \multirow{4}{*}{ Lake } & 10 & 0.617 & 0.886 & 0.896 & 0.906 \\
\hline & 20 & 0.481 & 0.784 & 0.808 & 0.819 \\
\hline & 30 & 0.402 & 0.719 & 0.741 & 0.755 \\
\hline & 40 & 0.376 & 0.662 & 0.688 & 0.701 \\
\hline \multirow{4}{*}{ HandXray } & 10 & 0.611 & 0.882 & 0.890 & 0.917 \\
\hline & 20 & 0.509 & 0.791 & 0.806 & 0.835 \\
\hline & 30 & 0.496 & 0.727 & 0.748 & 0.788 \\
\hline & 40 & 0.394 & 0.683 & 0.707 & 0.749 \\
\hline
\end{tabular}

\section{CONCLUTION}

In this paper, a robust functional minimization technique is presented for image enhancement while protecting the actual image details from the environmental disturbances. An efficient algorithm has been introduced and implemented under variational framework for finding the robust solutions. Moreover, the key challenges in order to edge and image details protection from high polishing affects have been addressed and tackled efficiently in cooperation with designated smoothing term. The performance of the proposed technique is also tested and verified by a number of sample experiments. The visual and quantitative results ensured the efficiency of the proposed technique in order to protect valuable image details while eliminating noise in homogeneous or edge regions than other methods.

\section{ACKNOWLEDGMENT}

This work was supported in part by the National Natural Science Foundation of China under Grants 61402290, 61472257, 61772343 and 61379030; in part by the Foundation for Distinguished Young Talents in Higher Education of Guangdong, China, under Grant 2014KQNCX134; in part by the Natural Science Foundation of Guangdong, China, under Grant 1714050003822; and in part by the Science Foundation of Shenzhen Science Technology and Innovation Commission, Grant JCYJ20160331114526190, China.

\section{REFERENCES}

[1] K. W. Hung and W. C. Siu, "Single-image superresolution using iterative Wiener filter based on nonlocal means," Signal Process. Image Commun., vol. 39, pp. 2645, Nov. 2015. 
[2] M. K. PK and M. M. Rahman, "Metal Artifact Reduction from Computed Tomography (CT) Images using Directional Restoration Filter," Int. J. Inf. Technol. Comput. Sci., vol. 6, no. 6, pp. 47-54, May 2014.

[3] X. Wang, H. Wang, J. Yang, and Y. Zhang, "A new method for nonlocal means image denoising using multiple images," PLoS One, vol. 11, no. 7, pp. 1-9, Jul. 2016.

[4] V. Fedorov and C. Ballester, "Affine Non-Local Means Image Denoising," IEEE Trans. Image Process., vol. 26, no. 5, pp. 2137-2148, May 2017.

[5] A. Li, D. Chen, K. Lin, and G. Sun, "Nonlocal Joint Regularizations Framework with Application to Image Denoising," Circuits, Syst. Signal Process., vol. 35, no. 8, pp. 2932-2942, Aug. 2016.

[6] X. Nie, H. Qiao, B. Zhang, and X. Huang, "A Nonlocal TV-Based Variational Method for PolSAR Data Speckle Reduction," IEEE Trans. Image Process., vol. 25, no. 6, pp. 2620-2634, Jun. 2016.

[7] W. Cheng and K. Hirakawa, "Minimum Risk Wavelet Shrinkage Operator for Poisson Image Denoising," IEEE Trans. Image Process., vol. 24, no. 5, pp. 1660-1671, May 2015.

[8] S. Gai, B. Zhang, C. Yang, and L. Yu, "Speckle noise reduction in medical ultrasound image using monogenic wavelet and Laplace mixture distribution," Digit. Signal Process. A Rev. J., vol. 72, pp. 192-207, Jan. 2018.

[9] A. Li, D. Chen, G. Sun, and K. Lin, "Sparse representation-based image restoration via nonlocal supervised coding," Opt. Rev., vol. 23, no. 5, pp. 776-783, Oct. 2016.

[10] T. Huang, W. Dong, X. Xie, G. Shi, and X. Bai, "Mixed noise removal via laplacian scale mixture modeling and nonlocal low-rank approximation," IEEE Trans. Image Process., vol. 26, no. 7, pp. 3171-3186, Jul. 2017.

[11] K. S. Zhang, L. Zhong, and X. Y. Zhang, "Image Restoration via Group 12,1 Norm-Based Structural Sparse Representation,” Int. J. Pattern Recognit. Artif. Intell., vol. 32, no. 04, p. 1854008, Apr. 2018.

[12] E. Ehsaeyan, "A new shearlet hybrid method for image denoising," Iran. J. Electr. Electron. Eng., vol. 12, no. 2, pp. 97-104, 2016.

[13] H. Al-Marzouqi and G. AlRegib, "Curvelet transform with learning-based tiling," Signal Process. Image Commun., vol. 53, pp. 24-39, Apr. 2017.

[14] G. Baloch and H. Ozkaramanli, "Image denoising via correlation-based sparse representation," Signal, Image Video Process., vol. 11, no. 8, pp. 1501-1508, May 2017.

[15] K. Mechlem, S. Allner, K. Mei, F. Pfeiffer, and P. B. Noël, "Dictionary-based image denoising for dual energy computed tomography," 2016, p. 97830E.

[16] Liu, "Image denoising via adaptive soft-thresholding based on non-local samples," in Proceedings of the IEEE Computer Society Conference on Computer Vision and Pattern Recognition, 2015, vol. 07-12-June, pp. 484-492.

[17] X. Xu and T. Bu, "An Adaptive Parameter Choosing Approach for Regularization Model," Int. J. Pattern Recognit. Artif. Intell., vol. 32, no. 08, p. 1859013, Aug. 2018.

[18] T. Veerakumar, R. P. K. Jagannath, B. N. Subudhi, and S. Esakkirajan, "Impulse Noise Removal Using Adaptive Radial Basis Function Interpolation," Circuits, Syst. Signal Process., vol. 36, no. 3, pp. 1192-1223, Mar. 2017.

[19] H. A. Alnabriss and I. S. I. Abuhaiba, "Improved Image Retrieval with Color and Angle Representation," Int. J. Inf. Technol. Comput. Sci., vol. 6, no. 6, pp. 68-81, May 2014.

[20] G. Aubert and P. Kornprobst, Mathematical problems in image processing: partial differential equations ... Springer, 2001

[21] D. Wang and J. Gao, "An improved noise removal model based on nonlinear fourth-order partial differential equations," Int. J. Comput. Math., vol. 93, no. 6, pp. 942954, Jun. 2016.

[22] X. Y. Liu, C. H. Lai, and K. A. Pericleous, "A fourthorder partial differential equation denoising model with an adaptive relaxation method," Int. J. Comput. Math., vol. 92, no. 3, pp. 608-622, Mar. 2015.

[23] Z. Tu, W. Xie, J. Cao, C. van Gemeren, R. Poppe, and R. C. Veltkamp, "Variational method for joint optical flow estimation and edge-aware image restoration," Pattern Recognit., vol. 65, pp. 11-25, May 2017.

[24] Y. Han, C. Xu, and G. Baciu, "A variational based smart segmentation model for speckled images," Neurocomputing, vol. 178, pp. 62-70, 2016.

[25] Y. Han, C. Xu, G. Baciu, M. Li, and M. R. Islam, "Cartoon and Texture Decomposition-Based Color Transfer for Fabric Images," IEEE Trans. Multimed., vol. 19, no. 1, pp. 80-92, Jan. 2017.

[26] P. Liu, L. Xiao, and J. Zhang, "A fast higher degree total variation minimization method for image restoration," Int. J. Comput. Math., vol. 93, no. 8, pp. 1383-1404, Aug. 2016.

[27] Y. Han, C. Xu, G. Baciu, and X. Feng, "Multiplicative noise removal combining a total variation regularizer and a nonconvex regularizer," Int. J. Comput. Math., vol. 91, no. 10, pp. 2243-2259, Oct. 2014.

[28] V. B. S. Prasath, D. Vorotnikov, R. Pelapur, S. Jose, G. Seetharaman, and K. Palaniappan, "Multiscale tikhonovtotal variation image restoration using spatially varying edge coherence exponent," IEEE Trans. Image Process., vol. 24, no. 12, pp. 5220-5235, Dec. 2015.

[29] P. Getreuer, "Total Variation Inpainting using Split Bregman," Image Process. Line, vol. 2, pp. 147-157, May 2012.

[30] Y. Han, X. C. Feng, G. Baciu, and W. W. Wang, "Nonconvex sparse regularizer based speckle noise removal," Pattern Recognit., vol. 46, no. 3, pp. 989-1001, 2013.

[31] M. Nikolova, M. K. Ng, and C. P. Tam, "Fast nonconvex nonsmooth minimization methods for image restoration and reconstruction," IEEE Trans. Image Process., vol. 19, no. 12, pp. 3073-3088, Dec. 2010.

[32] Z. Zuo, W. Yang, X. Lan, L. Liu, J. Hu, and L. Yan, "Adaptive nonconvex nonsmooth regularization for image restoration based on spatial information," Circuits, Syst. Signal Process., vol. 33, no. 8, pp. 2549-2564, Aug. 2014.

[33] J. Mejia, B. Mederos, R. A. Mollineda, and L. O. Maynez, "Noise Reduction in Small Animal PET Images Using a Variational Non-Convex Functional," IEEE Trans. Nucl. Sci., vol. 63, no. 5, pp. 2577-2585, Oct. 2016.

[34] L. I. Rudin, S. Osher, and E. Fatemi, "Nonlinear total variation based noise removal algorithms," Phys. D Nonlinear Phenom., vol. 60, no. 1-4, pp. 259-268, 1992.

[35] A. Chambolle, "An Algorithm for Total Variation Minimization and Applications," J. Math. Imaging Vis., vol. 20, no. 1, pp. 89-97, Jan. 2004.

[36] H. Yu, L. Zhao, and H. Wang, "An efficient procedure for removing random-valued impulse noise in images," IEEE Signal Process. Lett., vol. 15, pp. 922-925, 2008.

[37] C. Sutour, J. F. Aujol, C. A. Deledalle, and J. P. Domenger, "Adaptive regularization of the NL-means for video denoising," 2014 IEEE Int. Conf. Image Process. ICIP 2014, vol. 23, no. 8, pp. 2704-2708, Aug. 2014.

[38] A. N. Tikhonov and V. I. A. Arsenin, Solutions of Ill- 
Posed Problems, 1st ed. Winston, 1977.

\section{Authors' Profiles}

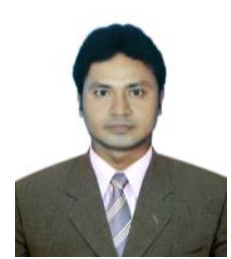

Md. Robiul Islam is a Ph.D. Fellow in Computer Science and Software Engineering, Shenzhen University, Guangdong, China. He received M.Sc. Engineering degree in Computer Science and Engineering from Khulna University of Engineering \& Technology (KUET), Bangladesh. He has several years teaching and administrative experiences in universities and organizations. His research interests include image processing, evolutionary and bio-inspired computing, machine learning, data mining and computer networking.

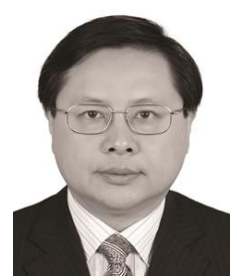

Xu Chen received the B.S. and M.S. degrees in Mathematics from Xidian University in 1986 and 1989 respectively, the Ph.D. degree in mathematics from Xi'an Jiaotong University in 1992. He is currently a Professor of Computational Science and Mathematics and Advisor for doctor students at Shenzhen University, Shenzhen, China. His research fields are information and computational science, image analysis and application of wavelet.

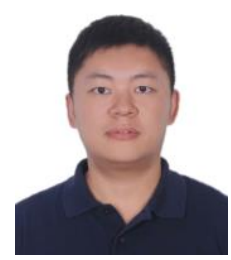

Han Yu received the Ph.D. degree in Mathematics from Xidian University, Xian, China in 2013. He is currently a Lecturer in College of Mathematics and Statistics, Shenzhen University, Shenzhen, China. He was a research associate in the GAMA Lab, Department of Computing, the Hong Kong Polytechnic University, Hong Kong, from Dec. 2011 to Oct. 2015. His research interests include variational and partial differential equation based image processing.

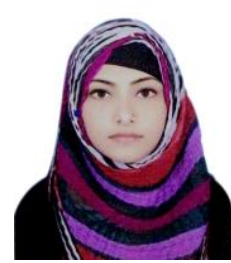

Sanjida Sultana Putul is currently pursuing B.Sc. Engineering in Computer Science and Engineering, North Western University, Khulna, Bangladesh. Her research interests include image processing, pattern recognition, data mining, machine learning, neural networks, and computer networking.

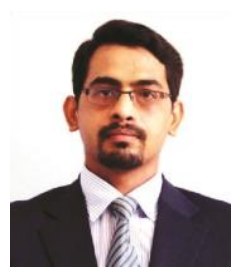

Rana Aamir Raza received the Ph.D. degree from Shenzhen University; he obtained his Master of Computer Science degree from Blekinge Tekniska Hogskola (BTH), Karlskrona, Sweden. He also received his M.Sc. degrees in Computer Science from Bahauddin Zakariya University Multan, Pakistan. Since 2010, he is working as an Assistant Professor in Department of Computer Science, Bahauddin Zakariya University Multan, Pakistan. His main research interests include pattern recognition, machine learning, neural networks, and their related applications.
How to cite this paper: Robiul Islam, Chen $\mathrm{Xu}, \mathrm{Yu}$ Han, Sanjida Sultana Putul, Rana Aamir Raza, "A Robust Functional Minimization Technique to Protect Image Details from Disturbances", International Journal of Information Technology and Computer Science(IJITCS), Vol.11, No.7, pp.1-8, 2019. DOI: $10.5815 /$ ijitcs.2019.07.01 